\title{
Robust Mixed Helices Promoted by 'Alternating Chirality' in a New Family of C-Linked Carbo- $\beta$-Peptides
}

G V M Sharma*a, K Ravinder Reddy ${ }^{\mathrm{a}}$, Palakodety Radha Krishna ${ }^{\mathrm{a}}$, A Ravi Sankar ${ }^{\mathrm{b}}, \mathrm{K}^{\mathrm{a}}$ Narsimulu ${ }^{\mathrm{b}}$, S Kiran Kumar ${ }^{\text {b }}$ P Jayaprakash ${ }^{\mathrm{c}}$ B Jagannadh*c and A C Kunwar*b

\section{Indian Institute of Chemical Technology, Hyderabad 500 007, India}

Synthesis Of C-linked Carbo- $\beta$-peptides: The requisite monomer 1 was prepared by the aza-Michael addition with benzyl amine in the presence of tetra-n-butyl ammonium fluoride (TBAF) on the sugar derived $\alpha, \beta$-unsaturated esters, while a mixture of $\mathbf{1}$ and $\mathbf{2}$ was prepared by the Michael addition in the absence of TBAF. Deprotection of Boc group was achieved with trifluoro acetic acid in $\mathrm{CH}_{2} \mathrm{Cl}_{2}$, while esters were hydrolyzed with $4 \mathrm{~N} \mathrm{NaOH}$ solution in $\mathrm{MeOH}$. Peptide coupling reactions were carried out in the presence of EDCI, $\mathrm{HOBt}$ and DIPEA in $\mathrm{CH}_{2} \mathrm{Cl}_{2}$.

All the compounds reported were purified by column chromatography over silica gel (60-120 mesh; EtOAc-Pet.Ether upto tri peptides; $\mathrm{MeOH}-\mathrm{CHCl}_{3}$ for tetra and hexa peptides).

Circular Dichroism Spectroscopy: CD spectra were acquired using JASCO-715 or JASCO-500 spectrometers at room temperature in $\mathrm{MeOH}$, using a 1-mm pathlength $\mathrm{CD}$ cell. Spectra represent the average of 3 scans (100 ms time constant, 2-nm bandwidth) and were background corrected and smoothened over 2 data points. Supporting Figures 1 and 2 illustrates the mean residue ellipticity of peptides $\mathbf{3 - 5}$ and 7-8, scanned from $260 \mathrm{nM}$ to $190 \mathrm{nM}$, at $100 \mu \mathrm{M}$ concentration.

\section{Supporting Figure 1. CD Spectra of $\mathbf{3 , 4}$ and $\mathbf{5}$}

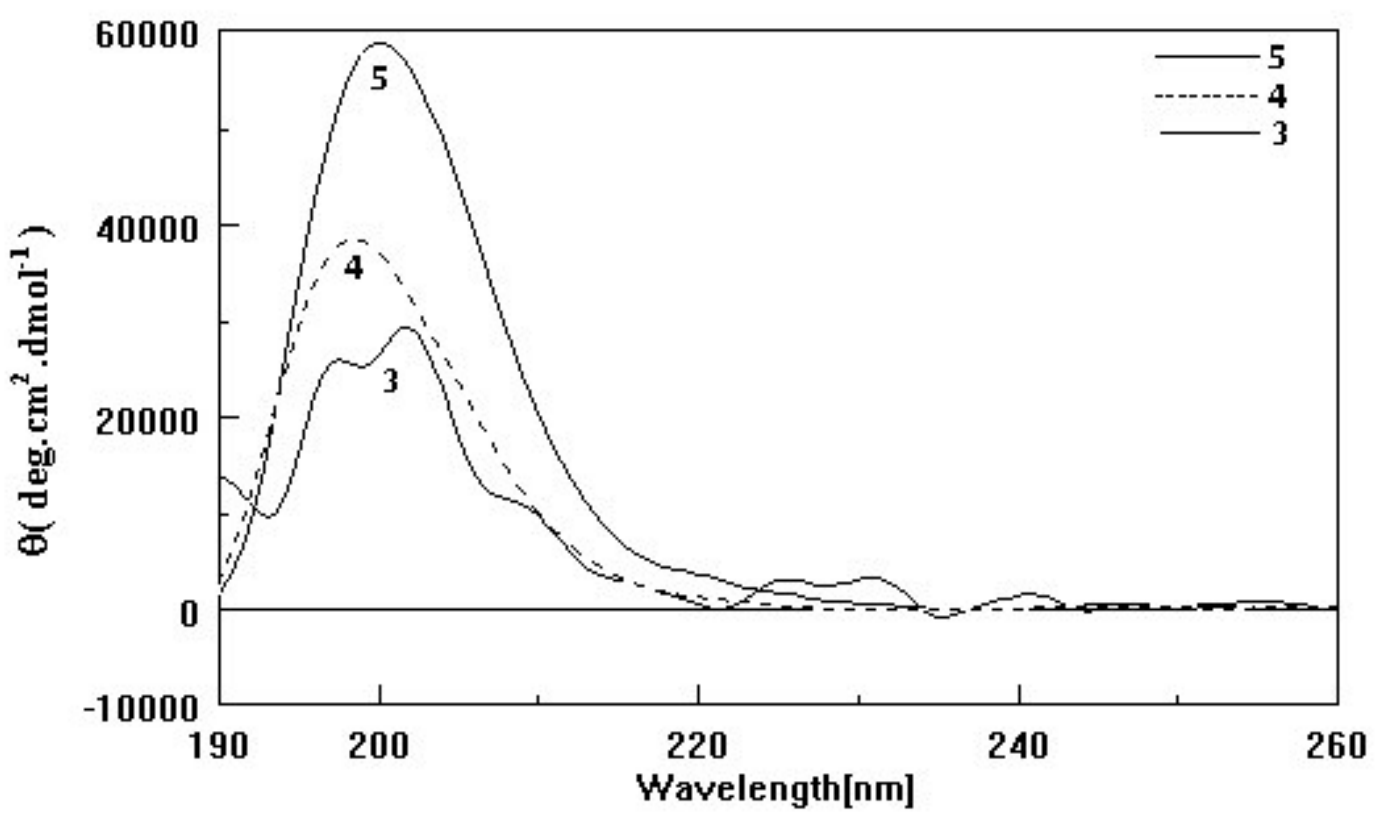

Page S-01 


\section{Supporting Figure 2. CD Spectra of $\mathbf{7}$ and $\mathbf{8}$}

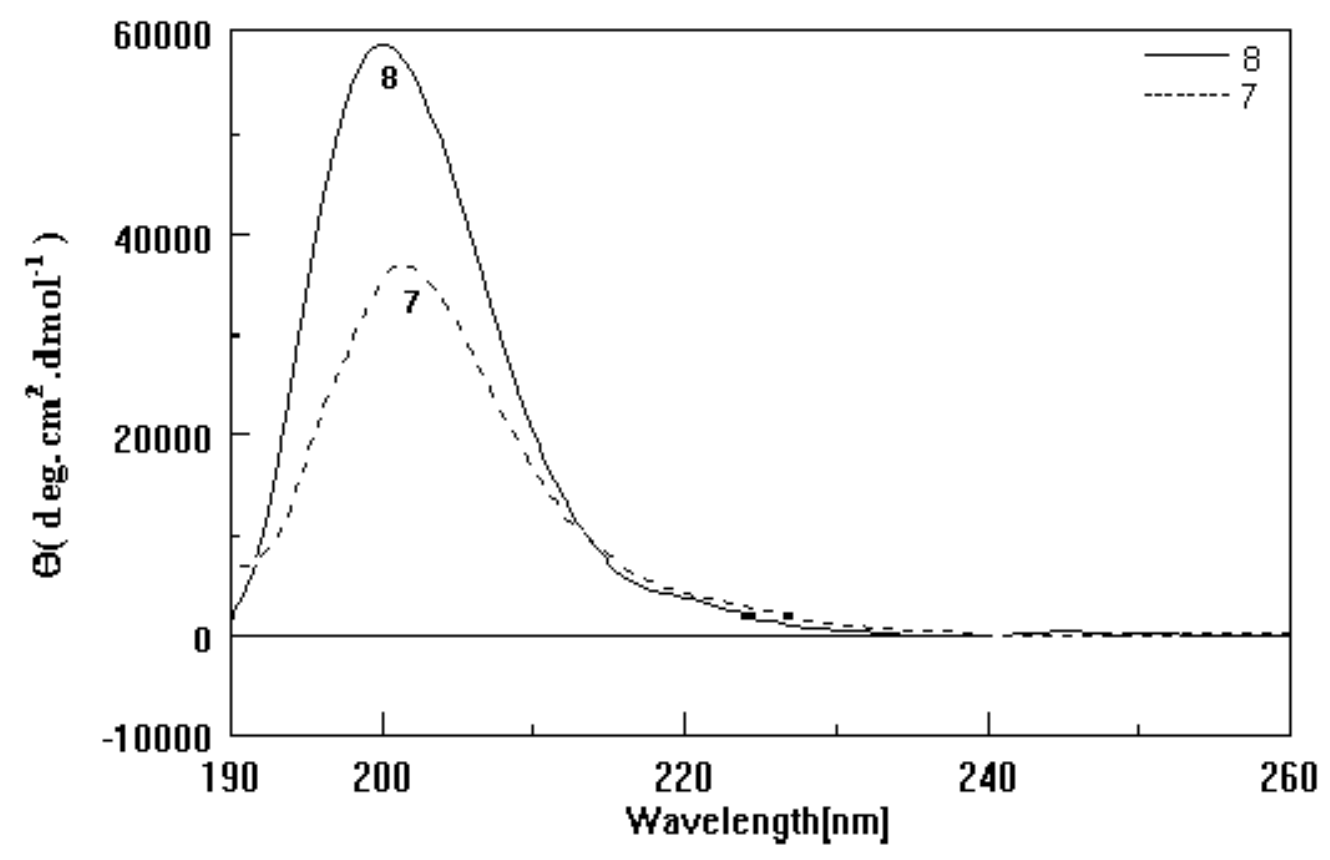

NMR Spectroscopy: NMR spectra were recorded on Varian Unity Inova - $500 \mathrm{MHz}$ and Bruker Avance $800 \mathrm{MHz}$ spectrometers at $303 \mathrm{~K}$ and $295 \mathrm{~K}$ respectively, with 7-10 mM solutions in appropriate solvents using tetramethylsilane as internal standard or the solvent signals as secondary standards, and the chemical shifts are shown in $\delta$ scales. Multiplicities of NMR signals are designated as s (singlet), d (doublet), t (triplet), q (quartet), br (broad), m (multiplet, for unresolved lines), etc. Two dimensional total correlation spectroscopy (TOCSY) and rotating frame nuclear Overhauser effect spectroscopy (ROESY) experiments were carried out. All the experiments were carried out in the phase-sensitive mode using the procedure of States et al. The spectra were acquired with 2 × 256 or 2 x 192 free induction decays (FID) containing 8-16 transients with relaxation delays of 1.0 to $2.0 \mathrm{~s}$. The ROESY experiments were performed with mixing time of 0.2 to $0.3 \mathrm{~s}$. For ROESY experiments a spin locking field of about $2 \mathrm{kHz}$ and pulsed field locking with $30^{\circ}$ pulses were used. The TOCSY experiments were performed with the spin locking field of about $10 \mathrm{kHz}$ and a mixing time of $0.08 \mathrm{~s}$. The two dimensional data were processed with Gaussian apodization in both the dimensions. In these oligomers the $\mathrm{N}$-terminal amide proton was easily assigned as it shows nOe with intra residue $\mathrm{C \alpha H}$ proton only, where as the other amide protons shows both intra residue and inter residue $\mathrm{NH} / \mathrm{C} \alpha \mathrm{H}$ nOe. This assignment has further supported from the nOe between the amide and Boc group. Similarly the $\mathrm{C} \alpha \mathrm{H}$ of the $\mathrm{C}$ - terminal residue has been identified from the presence of nOe only with the intra residue NH. The spectra (One Dimensional, TOCSY and ROESY) and Solvent titration studies plot are illustrated in the Supporting Figures 3-23 and the chemical shifts, coupling constants are given in Supporting Tables 1-5. 
Supporting Figure S-03: ${ }^{1} \mathrm{H}$ NMR Spectrum of $\mathbf{3}(500 \mathrm{MHz}, 303 \mathrm{~K}, \mathrm{CDCl} 3)$

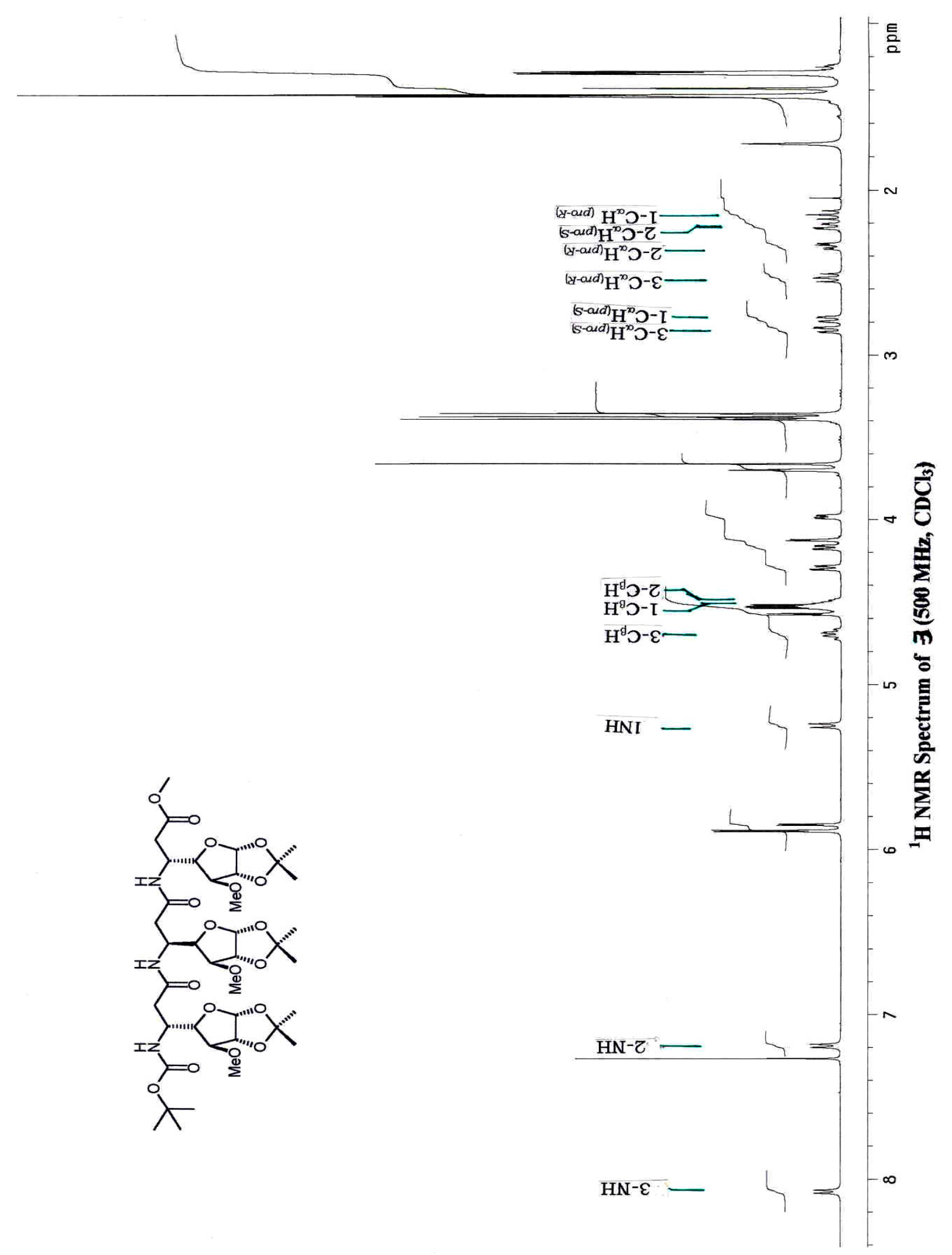

Page S-03 
Supporting Figure S-04: 2D-TOCSY Spectrum of 3 (500 MHZ, Temp-303K, CDCl3)

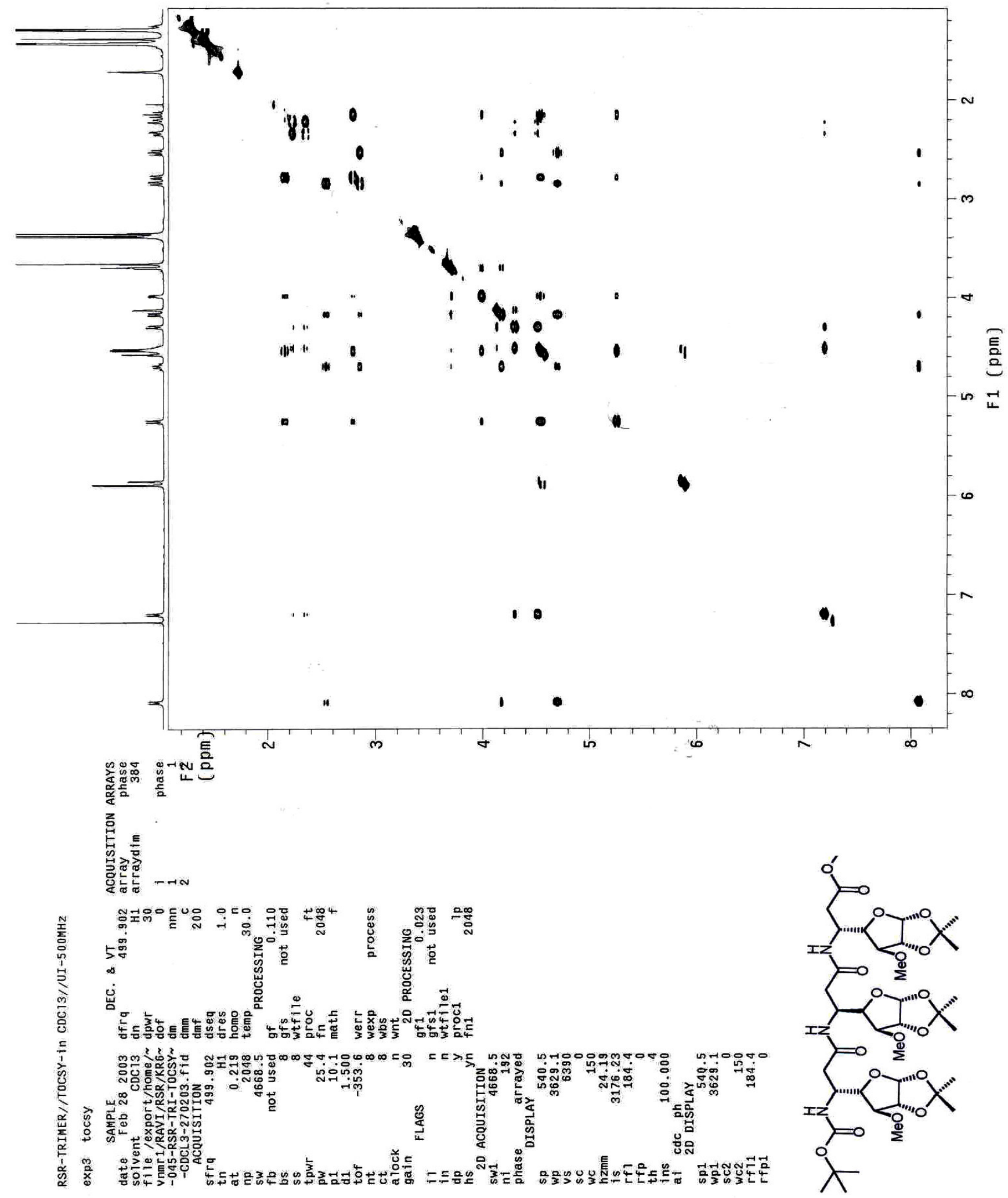

Page S-04 
Supporting Figure S-05: 2D- ROESY Spectrum of 3 (500 MHz, 303K, CDCl3)

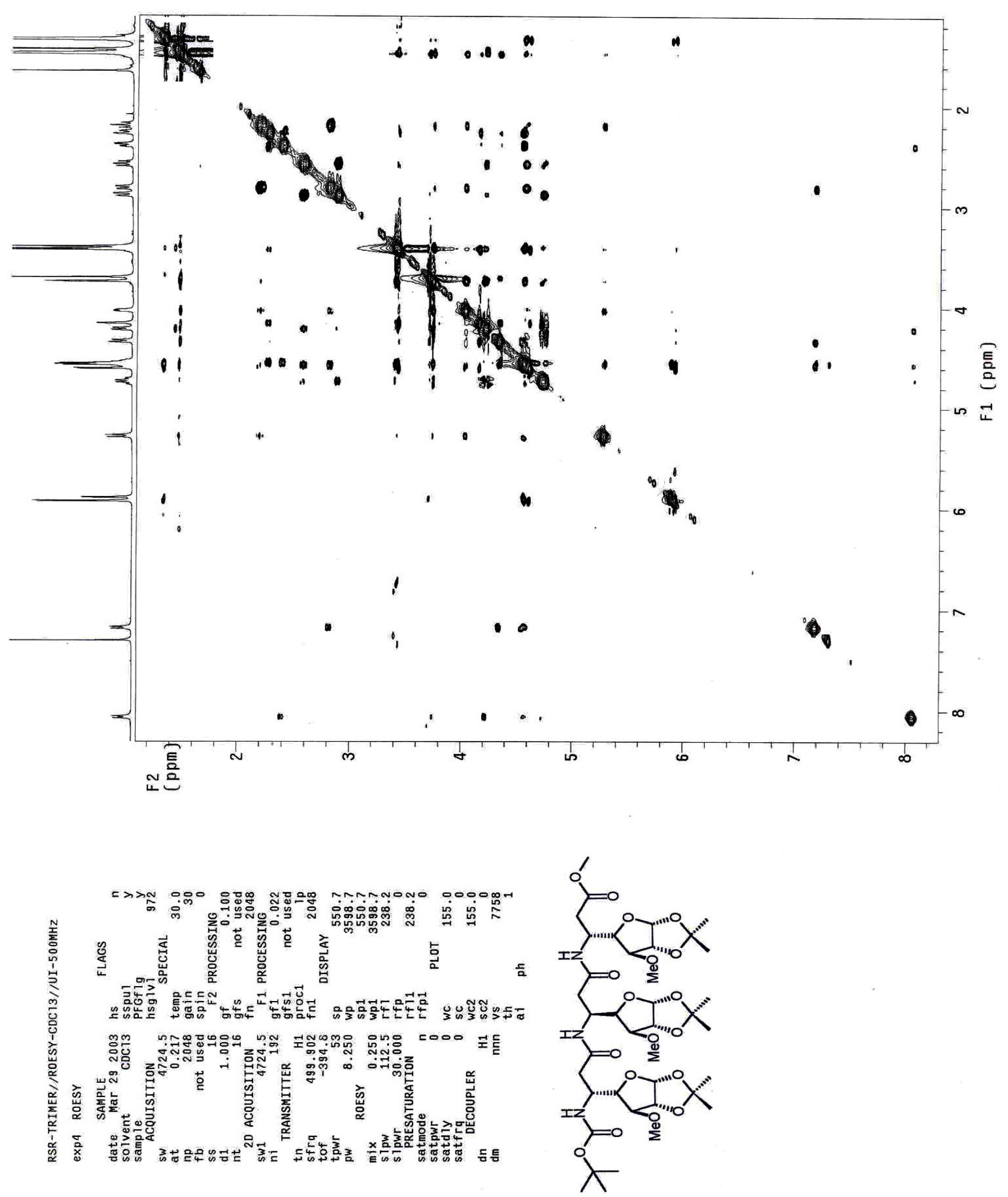

Page S-05 
Supporting Figure S-06: Solvent Titration Studies Plot for $\mathbf{3}$

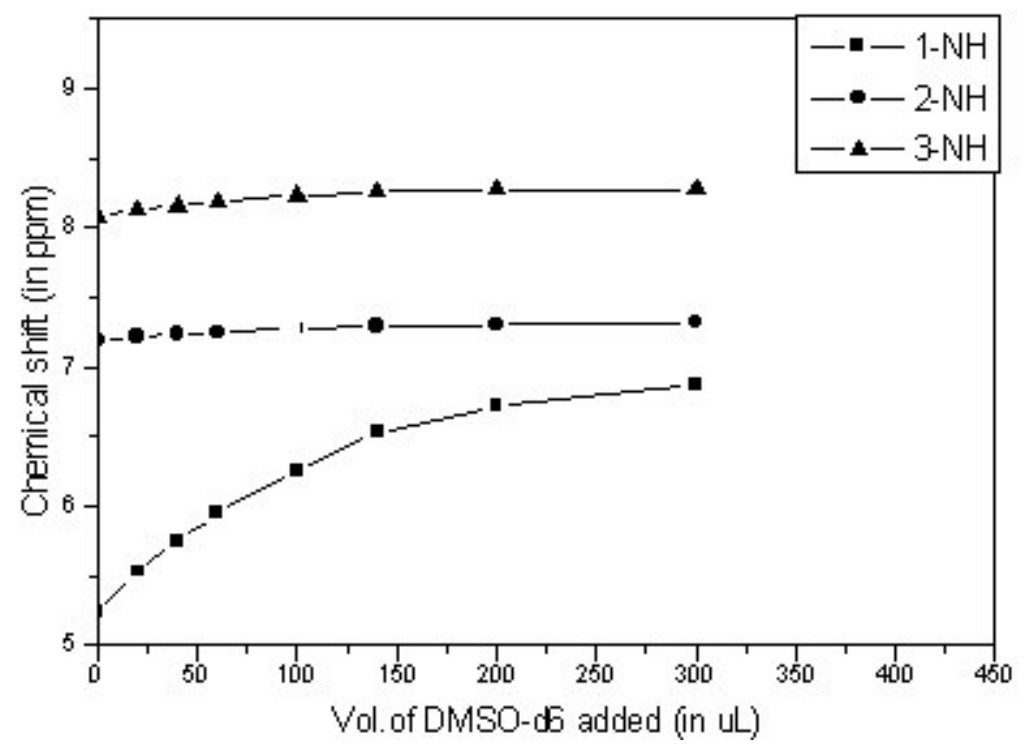

Supporting Table S-01:Chemical Shifts and Coupling Constants for 3 in $\mathrm{CDCl}_{3}(500 \mathrm{MHz})$.

\begin{tabular}{|c|c|c|c|}
\hline $\begin{array}{l}\text { Residue } \\
\text { Proton }\end{array}$ & 1 & 2 & 3 \\
\hline $\mathrm{NH}$ & $\begin{array}{l}5.26(\mathrm{~d}) \\
J=10.5\end{array}$ & $\begin{array}{r}7.19(\mathrm{~d}) \\
J=9.6\end{array}$ & $\begin{array}{r}8.08(\mathrm{~d}) \\
J=9.5\end{array}$ \\
\hline $\mathrm{C}_{\alpha} \mathrm{H}_{\text {(Pro-R) }}$ & $\begin{array}{l}2.16(\mathrm{dd}) \\
J=12.4,12.9\end{array}$ & $\begin{array}{c}2.34(\mathrm{dd}) \\
J=4.9,13.1\end{array}$ & $\begin{array}{c}2.53(\mathrm{dd}) \\
J=10.7,13.0\end{array}$ \\
\hline $\mathrm{C}_{\alpha} \mathrm{H}_{\text {(Pro-S) }}$ & $\begin{array}{l}2.78(\mathrm{dd}) \\
J=2.7,12.9\end{array}$ & $\begin{array}{c}2.22(\mathrm{dd}) \\
J=3.3,13.1\end{array}$ & $\begin{array}{r}2.85(\mathrm{dd}) \\
J=3.0,13.0\end{array}$ \\
\hline $\mathrm{C}_{\beta} \mathrm{H}$ & $4.53(\mathrm{~m})$ & $4.50(\mathrm{~m})$ & $4.69(\mathrm{~m})$ \\
\hline $\mathrm{C}_{4} \mathrm{H}$ & $\begin{array}{r}3.99(\mathrm{dd}) \\
J=3.1,7.3\end{array}$ & $\begin{array}{r}4.30(\mathrm{dd}) \\
J=3.0,9.8\end{array}$ & $\begin{array}{r}4.17(\mathrm{dd}) \\
J=3.1,9.7\end{array}$ \\
\hline $\mathrm{C}_{3} \mathrm{H}$ & $\begin{array}{c}3.71(\mathrm{~d}) \\
J=3.1\end{array}$ & $\begin{array}{c}4.13(\mathrm{~d}) \\
J=3.0\end{array}$ & $\begin{array}{l}3.70(\mathrm{~d}) \\
J=3.1\end{array}$ \\
\hline $\mathrm{C}_{2} \mathrm{H}$ & $\begin{array}{l}4.52(\mathrm{~d}) \\
J=4.0\end{array}$ & $\begin{array}{c}4.57(\mathrm{~d}) \\
J=3.9\end{array}$ & $\begin{array}{l}4.53(\mathrm{~d}) \\
J=3.9\end{array}$ \\
\hline $\mathrm{C}_{1} \mathrm{H}$ & $\begin{array}{l}5.85(\mathrm{~d}) \\
J=4.0\end{array}$ & $\begin{array}{l}5.89(\mathrm{~d}) \\
J=3.9\end{array}$ & $\begin{array}{c}5.89(\mathrm{~d}) \\
J=3.9\end{array}$ \\
\hline $\mathrm{OMe}$ & $3.39(\mathrm{~s})$ & $3.38(\mathrm{~s})$ & $3.36(\mathrm{~s})$ \\
\hline
\end{tabular}

Others: 3.66 (s,-CO OMe), 1.43 (s, Boc), 1.44, 1.44, 1.39, 1.306, 1.298, 1.29 (s, Acetonide $\mathrm{CH}_{3}$ 's). 
Supporting Figure S-07: ${ }^{1} \mathrm{H}$ NMR Spectrum of $4(500 \mathrm{MHz}, 303 \mathrm{~K}, \mathrm{CDCl} 3)$

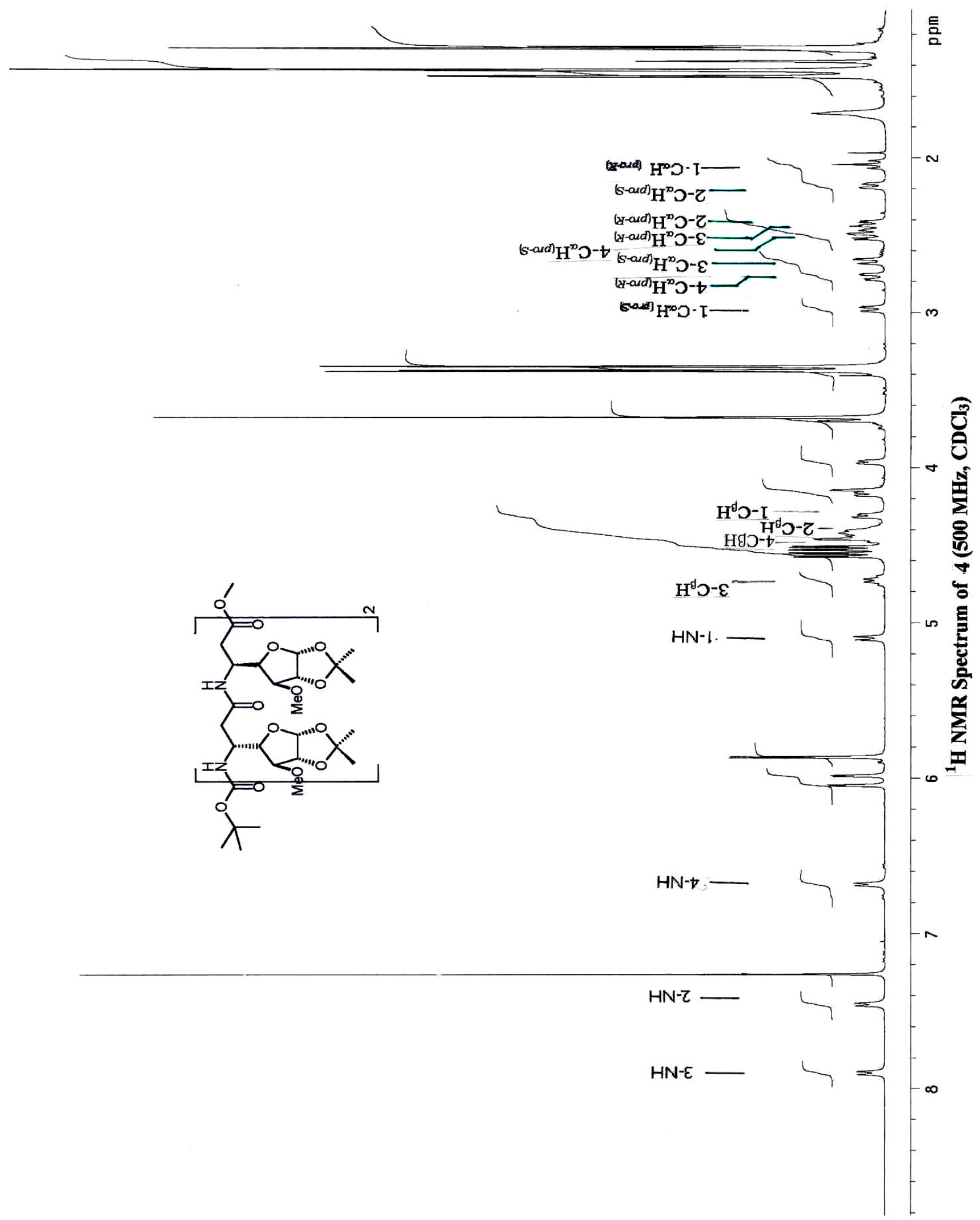


Supporting Figure S-08: 2D-TOCSY Spectrum of 4 (500 MHz, 303K, CDCl3)
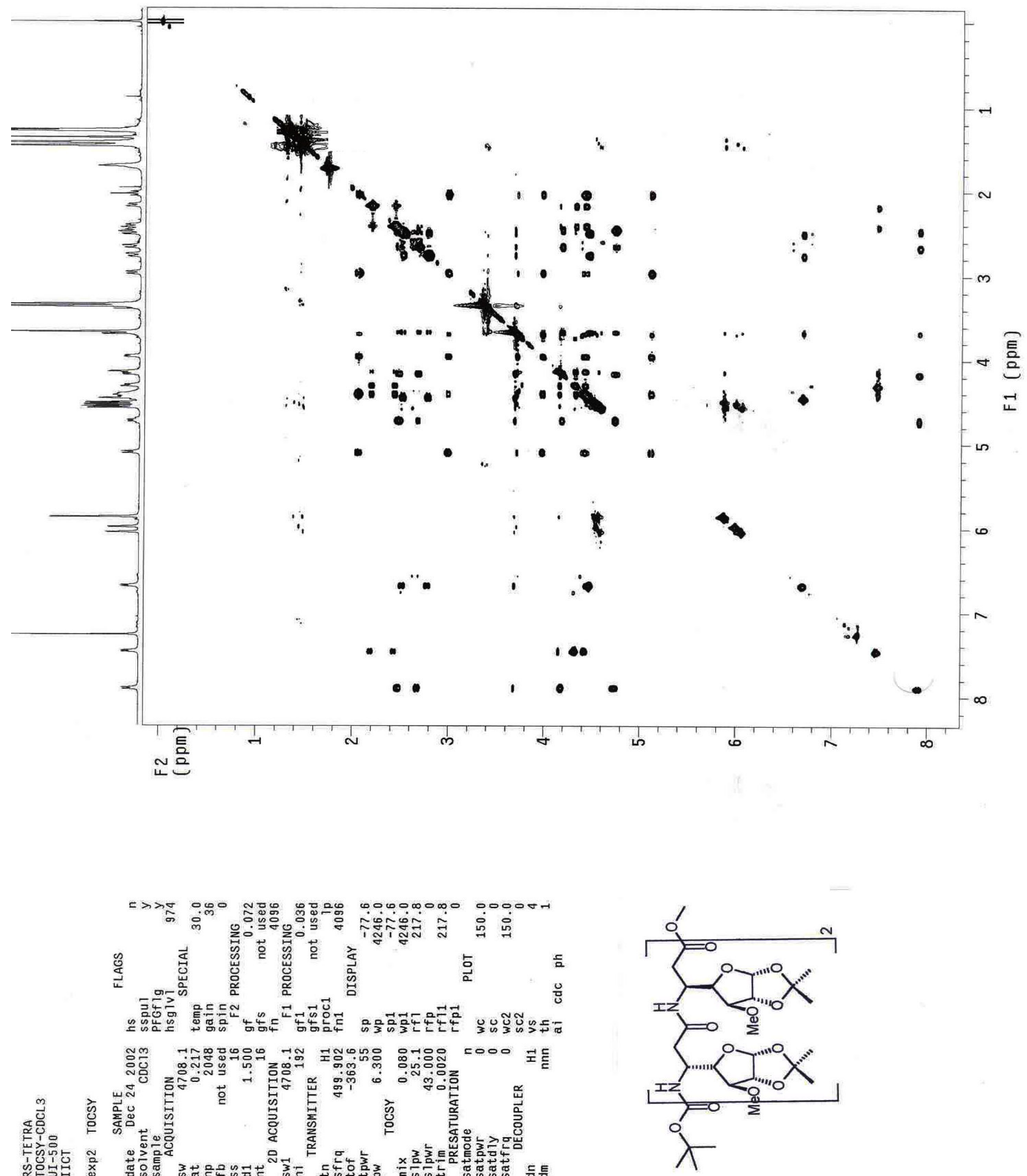

Page S-08 
Supporting Figure S-09: 2D-ROESY Spectrum of 4 (500 MHz, 303 K, CDCl3)
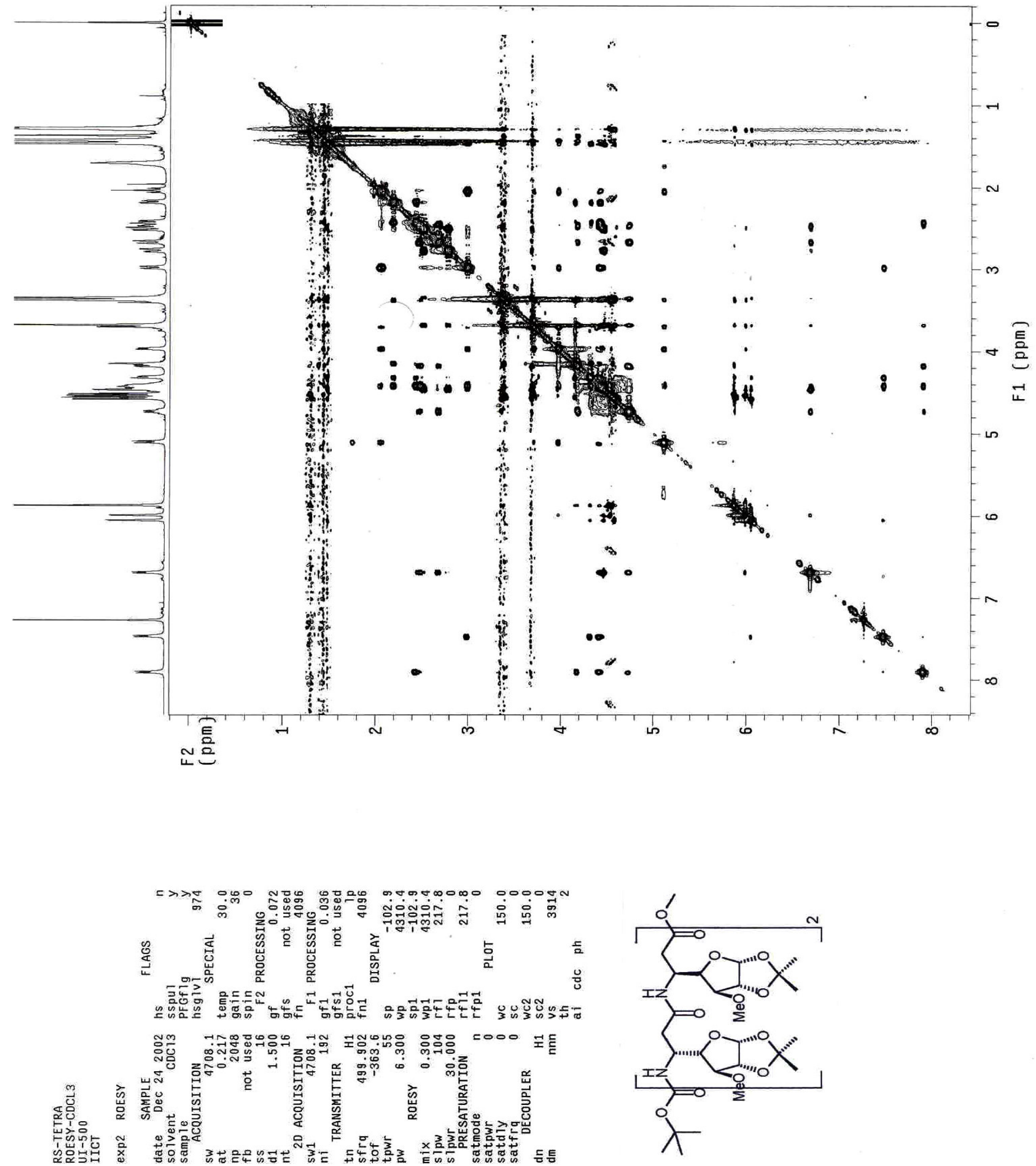
Supporting Figure S-10: Solvent Titration Studies Plot for 4

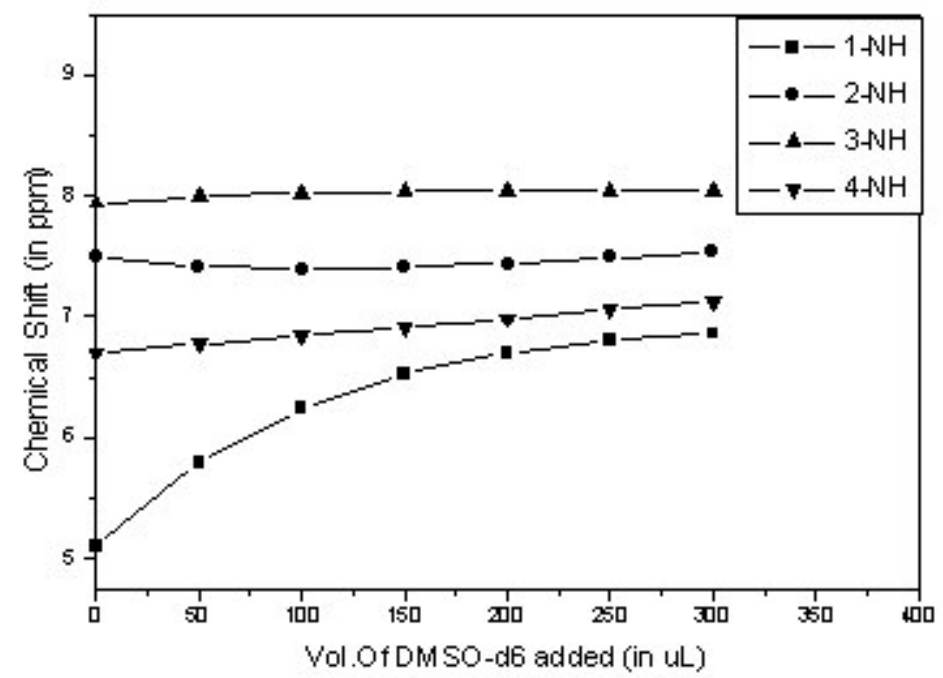

Supporting Table S-02: Chemical Shifts and Coupling Constants for 4 in $\mathrm{CDCl}_{3}(500 \mathrm{MHz})$.

\begin{tabular}{|c|c|c|c|c|}
\hline Protons & 1 & 2 & 3 & 4 \\
\hline $\mathrm{NH}$ & $\begin{array}{c}5.10(\mathrm{~d}) \\
J=10.6\end{array}$ & $\begin{array}{l}7.46(\mathrm{~d}) \\
J=8.5\end{array}$ & $\begin{array}{l}7.89(\mathrm{~d}) \\
J=9.6\end{array}$ & $\begin{array}{c}6.68(\mathrm{~d}) \\
J=8.2\end{array}$ \\
\hline $\mathrm{C}_{\alpha} \mathrm{H}_{(\text {Pro-R })}$ & $\begin{array}{l}2.04(\mathrm{t}) \\
J=13.0\end{array}$ & $\begin{array}{l}2.42(\mathrm{dd}) \\
J=4.7,13.2\end{array}$ & $\begin{array}{l}2.47(\mathrm{dd}) \\
J=10.5,14.8\end{array}$ & $\begin{array}{c}2.77(\mathrm{dd}) \\
J=4.4,15.5\end{array}$ \\
\hline $\mathrm{C}_{\alpha} \mathrm{H}_{\text {(Pro-S) }}$ & $\begin{array}{c}2.97(\mathrm{dd}) \\
J=2.9,13.0\end{array}$ & $\begin{array}{c}2.18(\mathrm{dd}) \\
J=2.6,13.2\end{array}$ & $\begin{array}{l}2.67(\mathrm{dd}) \\
J=2.8,14.8\end{array}$ & $\begin{array}{r}2.50(\mathrm{dd}) \\
J=5.2,15.5\end{array}$ \\
\hline $\mathrm{C}_{\beta} \mathrm{H}$ & $4.40(\mathrm{~m})$ & $4.41(\mathrm{~m})$ & $4.72(\mathrm{~m})$ & $4.47(\mathrm{~m})$ \\
\hline $\mathrm{C}_{4} \mathrm{H}$ & $\begin{array}{c}3.96(\mathrm{dd}) \\
J=3.2,8.2\end{array}$ & $\begin{array}{l}4.31(\mathrm{dd}) \\
J=3.3,9.8\end{array}$ & $\begin{array}{l}4.16(\mathrm{dd}) \\
J=3.2,9.3\end{array}$ & $\begin{array}{c}4.46(\mathrm{dd}) \\
J=3.2,10.2\end{array}$ \\
\hline $\mathrm{C}_{3} \mathrm{H}$ & $\begin{array}{l}3.69(\mathrm{~d}) \\
J=3.2\end{array}$ & $\begin{array}{l}4.14(\mathrm{~d}) \\
J=3.3\end{array}$ & $\begin{array}{l}3.67(\mathrm{~d}) \\
J=3.2\end{array}$ & $\begin{array}{c}3.68(\mathrm{~d}) \\
J=3.2\end{array}$ \\
\hline $\mathrm{C}_{2} \mathrm{H}$ & $\begin{array}{c}4.53(\mathrm{~d}) \\
J=4.0\end{array}$ & $\begin{array}{c}4.55(\mathrm{~d}) \\
J=3.9\end{array}$ & $\begin{array}{l}4.51(\mathrm{~d}) \\
J=3.9\end{array}$ & $\begin{array}{r}4.57(\mathrm{~d}) \\
J=3.9\end{array}$ \\
\hline $\mathrm{C}_{1} \mathrm{H}$ & $\begin{array}{l}5.98(\mathrm{~d}) \\
J=4.0\end{array}$ & $\begin{array}{c}5.87(\mathrm{~d}) \\
J=3.9\end{array}$ & $\begin{array}{l}5.86(\mathrm{~d}) \\
J=3.9\end{array}$ & $\begin{array}{r}6.04(\mathrm{~d}) \\
J=3.9\end{array}$ \\
\hline $\mathrm{OMe}$ & $3.37(\mathrm{~s})$ & $3.33(\mathrm{~s})$ & $3.35(\mathrm{~s})$ & $3.34(\mathrm{~s})$ \\
\hline
\end{tabular}

Others: 3.67 (s, OMe), 1.43 (s, Boc), 1.48, 1.47, 1.43 (2), 1.38, 1.30, 1.29, 1.28 (s, Acetonide $\mathrm{CH}_{3}$ 's). 
Supporting Figure S-11: 1H NMR Spectrum of 5 (500 MHz, CDCl3)

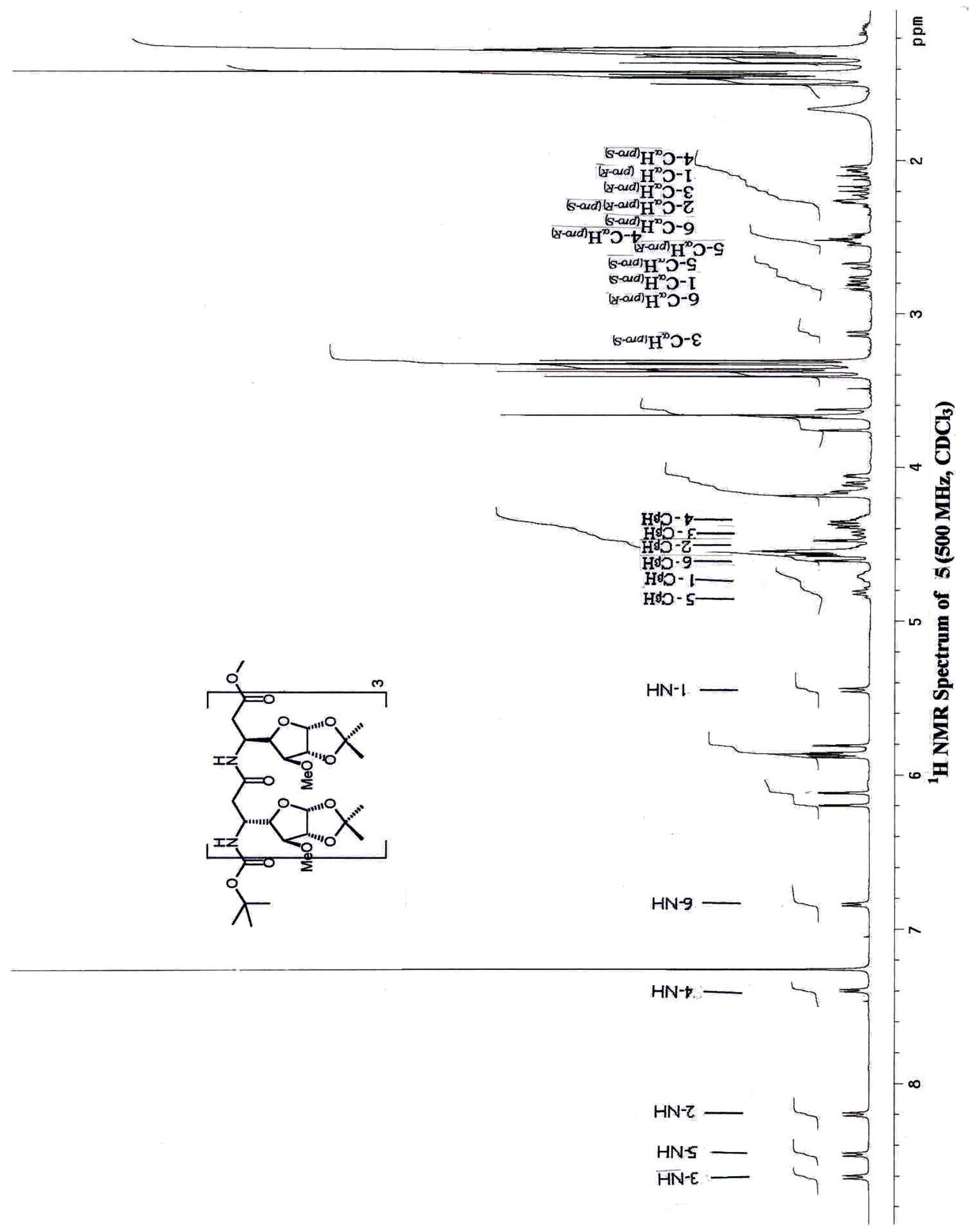

Page S-11 